\title{
General Discussion on Source Counts
}

Catherine Cesarsky: To launch the discussion about the models which we just heard, I would like to make three comments:

1. Fitting the infrared counts (especially the integral ones) does not establish the validity of a model. There is a degeneracy: widely different models can fit the integral counts. To obtain the real answer, it is necessary to understand the nature and the distances of the detected sources.

2. While giant ellipticals formed probably at high redshifts $z>3$, spheroid galaxies are being formed at much lower redshifts. This formation is accompanied by bursts of star formation and mid-infrared emission. Thus a detailed study of some ISOCAM galaxies may lead to a better understanding of the formation of spheroid galaxies and, by analogy, of giant ellipticals.

3. Normal and starburst galaxies are not two different populations but different steps during the evolution of the same population. It is valid to treat them as separate populations insofar as the starburst phenomena are decoupled from the optical evolution of the host galaxy. A large number of local, normal (not active or starburst) galaxies have been studied with ISOCAM at 7 and 15 microns; they don't appear to have excessive emission in the mid-infrared, and are unlikely to be candidates for distant $z \sim 0,8$ MIR sources.

Michael Rowan-Robinson: Isn't it rather surprising in Franceschini's scenario (in which the far infrared and submillimetre background is generated in highly opaque starbursts, with a truncated IMF), that the star formation history derived from the UV without correction for dust is so similar in shape to that derived from infrared and submillimetre data?

David Elbaz: The question of the similarity of the shape of the redshift evolution of the comoving density of star formation in the IR versus the UV may not be a relevant one if we compare the latest results of Cowie et al. for the UV to the models and data presented here for the IR, which are quite different: $L(z) \sim(1+z)^{1.9}$ in the UV is much flatter than the evolution observed in the IR.

Bernard Pagel: I think a total diffuse background of $60 \mathrm{nW} \mathrm{m}^{-2} \mathrm{sr}^{-1}$ is compatible with a universal Salpeter IMF. 